\title{
Characteristics and perspectives of families waiting to adopt a child with Down syndrome
}

\author{
Heidi L. Lindh, $M S^{1}$, Robin Steele, BA ${ }^{2}$, Jane Page-Steiner, $B A^{2}$, and Alan E. Donnenfeld, $M D^{3}$
}

\begin{abstract}
Purpose: The study's purpose was to obtain information on the characteristics and perspectives of families interested in adopting children with Down syndrome. Methods: A questionnaire-based survey was administered to individuals and families on a waiting list to adopt children with Down syndrome. Information on (1) demographic, (2) family structure, (3) Down syndrome exposure, (4) Down syndrome knowledge, (5) reasons for considering adoption, (6) adoption process, and (7) perspective on raising children with Down syndrome was assessed. Results: From 199 mailed surveys, there were 72 respondents (36.2\%) of whom six had previously adopted a child with Down syndrome. Forty-eight percent learned of the possibility of adopting children with Down syndrome through the Internet, whereas only one respondent obtained this information from a medical professional. The primary reasons for considering adoption were that prospective adoptive families were equipped with the necessary resources and had previous positive experiences with individuals who have Down syndrome. Conclusions: Many families are eager to adopt children with Down syndrome. Interest in this option stems from having resources to care for these children and previous positive experiences with individuals with Down syndrome. Information regarding adoption was rarely obtained from health care providers. When counseling pregnant women diagnosed with a Down syndrome fetus, adoption should be discussed so that all options regarding pregnancy management may be explored. Genet Med 2007:9(4):235-240.
\end{abstract}

Key Words: Adoption, Down syndrome, prenatal diagnosis

Adoption of children with special needs is a relatively new experience. In the 1950s, adoption was rarely considered an option if a child had mental retardation. ${ }^{1}$ By the early 1980s, however, the trend had shifted with regard to adoption of children with special needs. According to the National Committee for Adoption in 1985, 27.6\% of all unrelated adoptions in the United States involved special-needs children. ${ }^{2}$ By the mid1990 s, approximately half $(48.5 \%)$ of unrelated domestic adoptions involved children with disabilities. ${ }^{3}$

Since the first studies were published in the late 1960s indicating that midtrimester amniocentesis was possible for the purpose of detecting chromosomal aberrations including trisomy $21,4,5$ hundreds of thousands of women have undergone this procedure and many have been informed that their fetus has Down syndrome. Expectant couples are often counseled that they have two options: continuation or termination of the pregnancy. A third option is to continue the pregnancy and place the child with Down syndrome for adoption after birth.

From ${ }^{1}$ Genzyme Genetics, Dallas, Texas; ${ }^{2}$ Down syndrome Association of Greater Cincinnati, Cincinnati, Ohio; and ${ }^{3}$ Genzyme Genetics, Philadelphia, Pennsylvania.

Alan E. Donnenfeld, MD, Genzyme Genetics, 353 Lankenau Medical Building East, Wynnewood, PA 19096. E-mail: alan.donnenfeld@genzyme.com.

The mailing costs of this research study were provided by Genzyme Genetics.

Submitted for publication July 21, 2006.

Accepted for publication January 22, 2007.

DOI: 10.1097/GIM.0b013e31803d16d8
Until recently, adoption had not been traditionally discussed, because many professionals were unaware that families were available who desired to adopt children with special needs. ${ }^{6}$ Although this awareness has increased, many obstetricians are still not familiar with this possibility.

Limited information regarding the nature of families interested in the adoption of children with Down syndrome is available. One 1988 French study assessed the adoption and foster care of infants with Down syndrome. Their results indicated that approximately $19 \%$ of babies with Down syndrome who were born in the Paris and Marseilles regions between 1981 and 1990 were placed for adoption by their birth parents. However, only half of these children were ultimately adopted by a family. The remaining children were placed in foster care or an institution. $^{7}$

In England, one agency in the late 1980s reported that they had placed 35 children with Down syndrome for adoption within less than a decade. The investigators concluded that permanent family placement of children with Down syndrome was not only realistic, but there are generally enough families available to "choose" from to find an appropriate home for any given child. ${ }^{8}$

Each day parents receive the news that their fetus or newborn infant has Down syndrome. These individuals are faced with an enormous decision. If this information is determined during the pregnancy, the options are to continue the pregnancy and raise the child, continue the pregnancy and place the 
child for adoption, or terminate the pregnancy. If the diagnosis of Down syndrome is made at birth, the former two options are available. Presently, there is scant information regarding the availability and nature of families and individuals waiting to adopt children with Down syndrome. The aim of this study is to identify the characteristics of these families and individuals, as well as the reasons behind this decision. In addition, the present study hopes to facilitate increased awareness within the medical community of the option of adoption for fetuses identified with Down syndrome.

\section{MATERIALS AND METHODS}

A 40-question survey was developed and mailed to all 199 individuals and/or families who are currently on the waiting list to adopt a child through the Adoption Awareness Program of the Down Syndrome Association of Greater Cincinnati (DSAGC). This organization facilitates the adoption of children with Down syndrome through local adoption agencies. The DSAGC has been keeping a list of interested prospective adoptive parents for more than 20 years and has helped in facilitating more than 100 adoptions of children with Down syndrome.

The survey and a cover letter describing the purpose of the study, along with a stamped, self-addressed return envelope, were sent to each potential participant. Participants were asked to return the survey within 2 weeks of its receipt. Institutional review board exemption for this investigation was obtained from the DSAGC. The content of the questionnaire was also approved by the DSAGC.

The authors developed a questionnaire that used a quantitative approach and that addressed topics including demographic characteristics, adoption history, family structure, exposure to and knowledge of Down syndrome, opinions regarding Down syndrome, reasons for pursuing adoption of a child with Down syndrome, and adoption process characteristics.

Responses to each survey item were coded, labeled, and entered into a database. Data analysis and descriptive statistics were evaluated using the Statistical Package for the Social Sciences program (SPSS Inc., Chicago, IL), including numbers of respondents choosing a particular answer, frequencies, means, standard deviations, and ranges, where appropriate. Openended items were excluded from the statistical analyses but are addressed descriptively.

\section{RESULTS}

Of 199 surveys mailed, there were 72 respondents (36.2\%). Of the 72 respondents waiting to adopt, six had already adopted a child with Down syndrome through the DSAGC and were on the waiting list to adopt another child with this disorder. Ten families (14\%) had a previous biological child with Down syndrome. Just less than half (47.1\%) of the families had been waiting for 7 months to 5 years, with the most frequent response being 1 to 2 years (20\%).
Table 1

Demographic characteristics of individuals waiting to adopt a child with Down syndrome $(n=72)$

\begin{tabular}{lll}
\hline Characteristic & \multicolumn{2}{c}{ Response } \\
\hline Age & $\leq 40 \mathrm{y}$ & $(51.4 \%)$ \\
Race & White & $(84.3 \%)$ \\
Education & High school diploma & $(100 \%)$ \\
& College degree & $(54.2 \%)$ \\
Annual income & $>\$ 40,000$ & $(60.9 \%)$ \\
Marital status & Married & $(73.2 \%)$ \\
Religious affiliation & Christian & $(86.4 \%)$ \\
Hometown population & $\leq 50,000$ & $(68.2 \%)$ \\
Biological children & $\geq 1$ & $(66.2 \%)$ \\
Adopted children & $\geq 1$ & $(60.4 \%)$ \\
Child with Down syndrome & $\geq 1$ & $(50 \%)$ \\
Child with other disabilities & $\geq 1$ & $(59.7 \%)$ \\
\hline
\end{tabular}

Demographic characteristics of those 72 individuals and families currently waiting to adopt a child with Down syndrome are shown in Table 1. The mean age of respondents was 40 years (range $26-53$ years). All responders had a high school education, and $17 \%$ had obtained a master's degree. A religious affiliation was claimed by $92 \%$ of respondents, with the most common being Christian (86.4\%). Other religious affiliations included Jewish (4.5\%) and Buddhist (1.5\%). More than $80 \%$ of respondents resided in nonurban communities with populations less than 200,000. Seventy-three percent of respondents indicated they were married. Of those not married, $11.3 \%$ were single, $11.3 \%$ were divorced, $1.4 \%$ widowed, and $2.8 \%$ indicated "other" regarding marital status.

Figure 1 illustrates the sources by which respondents became aware of the possibility of adopting a child with Down syndrome. The most common source was the Internet (48\%), with only 1 respondent learning of this opportunity through their physician.

Approximately one third (34\%) of respondents currently on the waiting list had adopted a child with Down syndrome through an organization other than the DSAGC, and approximately half (49\%) had previously adopted a child/children with a disability other than Down syndrome. More than half (52\%) were also on another waiting list to adopt a child, and of those, $65 \%$ were on a list to specifically adopt a child with Down syndrome.

When respondents were asked if they would prefer adopting a child with Down syndrome versus a child without special needs, $67 \%$ stated that they would prefer to adopt a child with Down syndrome, and none of the respondents stated they would rather adopt a child without Down syndrome.

Sixty-six percent of respondents have their own biological children, and $70.4 \%$ indicated that they have one or more adopted children (mean 2.5). Half of the respondents did not have a biological or adopted child with Down syndrome; how- 


\section{Source of Awareness of Adoption Awareness Program $(n=89)$}

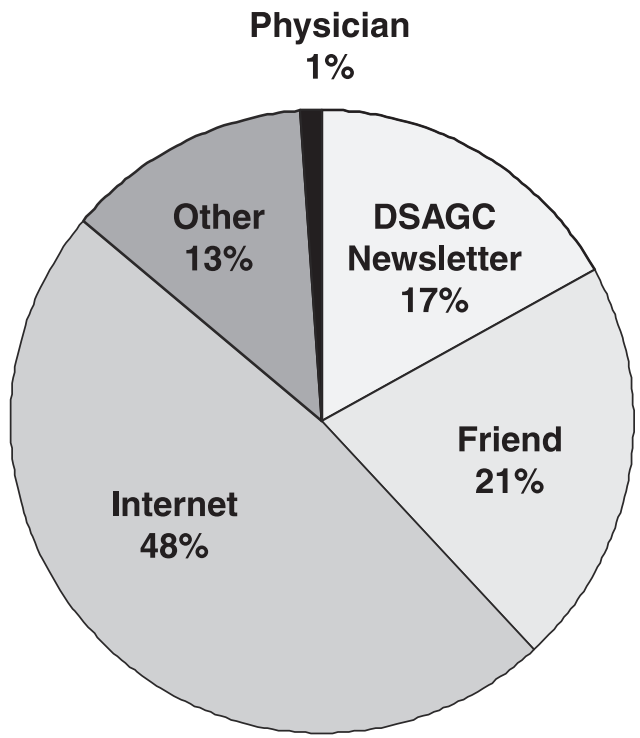

Fig. 1. Source of awareness of adoption awareness program. DSAGC, Down syndrome Association of Greater Cincinnati.

ever, $59.7 \%$ of respondents did have at least one child (biological or adopted) with a disability other than Down syndrome.

Figure 2 illustrates the personal experience and prior exposure to individuals with Down syndrome. Respondents were asked to indicate all that applied from a list of 11 possible means of exposure. The most common means of exposure to
Down syndrome individuals was "work with an individual with Down syndrome," which was indicated by $50 \%$ of respondents. Respondents with no personal knowledge of an individual with Down syndrome constituted $8.3 \%$ of all prospective adoptive parents.

The majority of the 72 respondents were well aware of the medical conditions associated with Down syndrome (Fig. 3). Respondents were asked to indicate which of the following are at an increased risk in individuals with Down syndrome: leukemia, thyroid problems, early-onset Alzheimer's disease, bowel function abnormalities, increased susceptibility to pneumonia, short stature, and visual impairment. As shown in Figure 3, all respondents were aware that individuals with Down syndrome have mental retardation, and 96\% stated that they were willing to adopt a child with Down syndrome without prior knowledge of their intelligence quotient. Seventy-five percent of respondents were willing to adopt a child with Down syndrome who had a cardiac defect.

Respondents were asked to choose from a list of seven reasons (including all that apply) that may be a part of their decision to adopt a child with Down syndrome. Respondents could also write in other reasons (Fig. 4) The most highly noted reason for choosing to adopt a child with Down syndrome was that the respondent felt equipped with the resources to raise a child with Down syndrome (85\%).

For those families and individuals who are currently waiting to adopt a child with Down syndrome, $88 \%$ strongly agree that there needs to be more awareness in the medical community regarding the availability of people waiting to adopt children with Down syndrome. The majority strongly agree that individuals with Down syndrome are an important part of our

\section{Exposure to Individuals with Down Syndrome $(n=72), D S=$ Down syndrome}

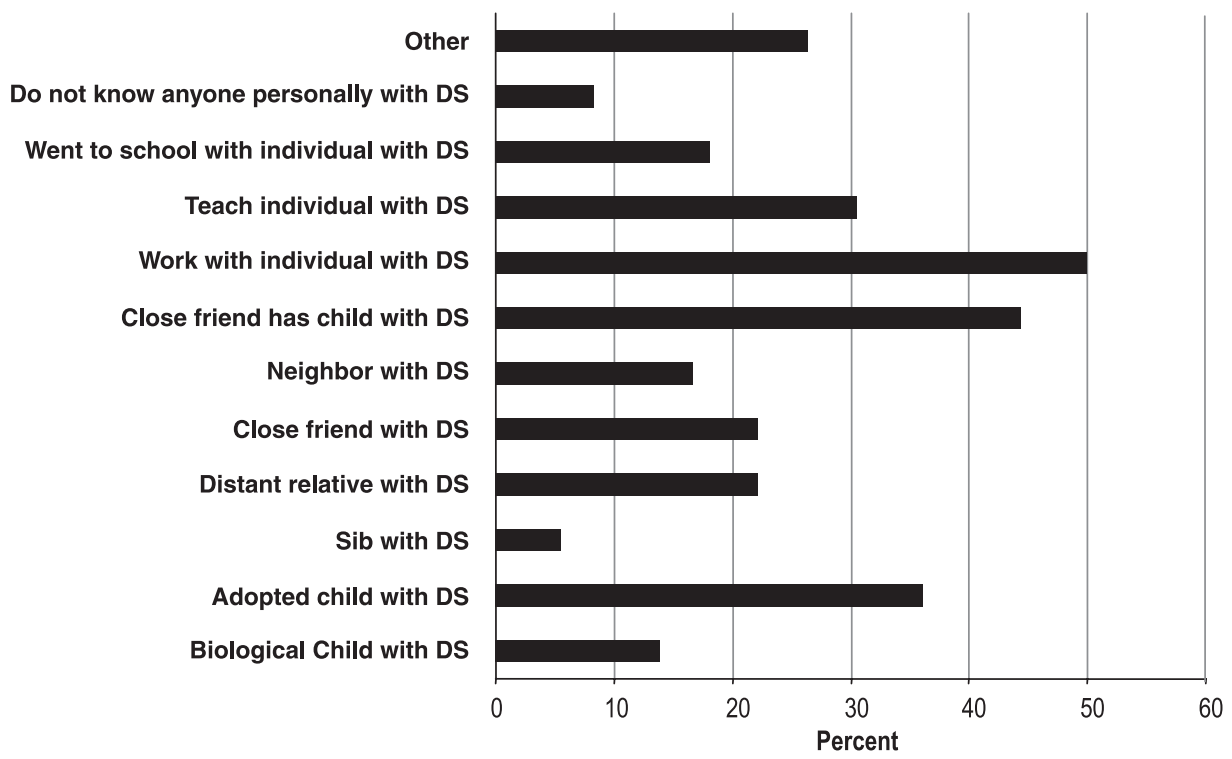

Fig. 2. Exposure to individuals with Down syndrome. DS, Down syndrome. 


\section{Awareness of medical conditions associated with Down syndrome $(n=72)$}

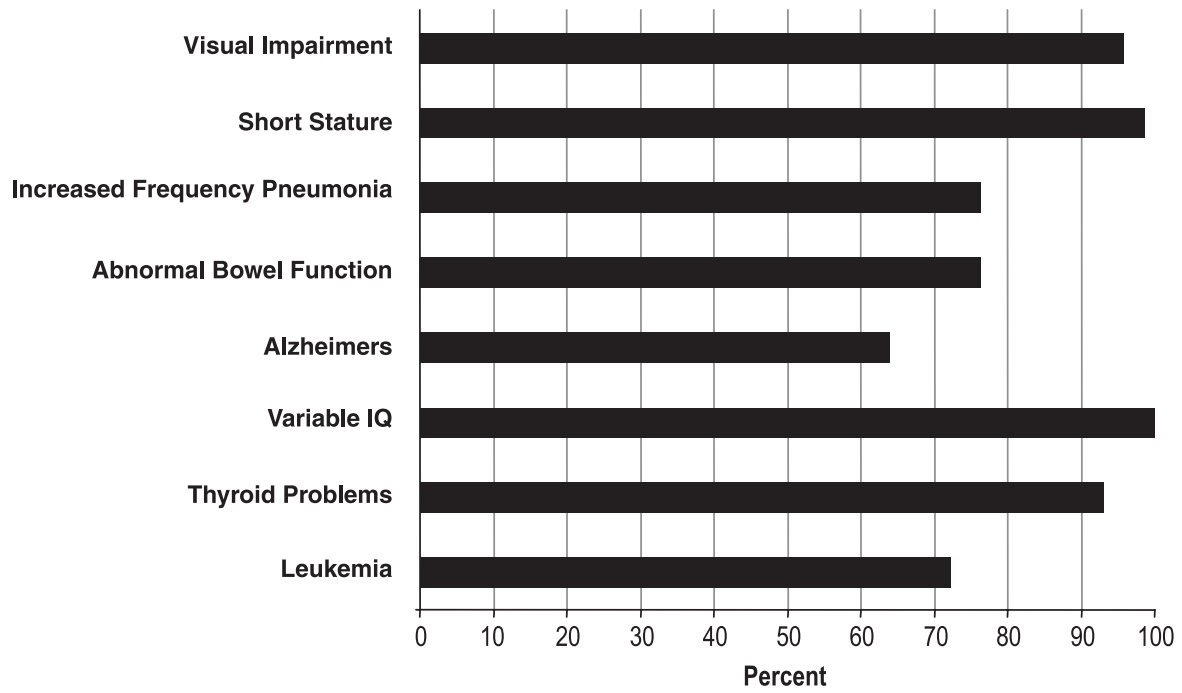

Fig. 3. Awareness of medical conditions associated with Down syndrome. IQ, intelligence quotient.

\section{Reasons People Are Interested in Adopting a Child with Down Syndrome $(n=72)$}

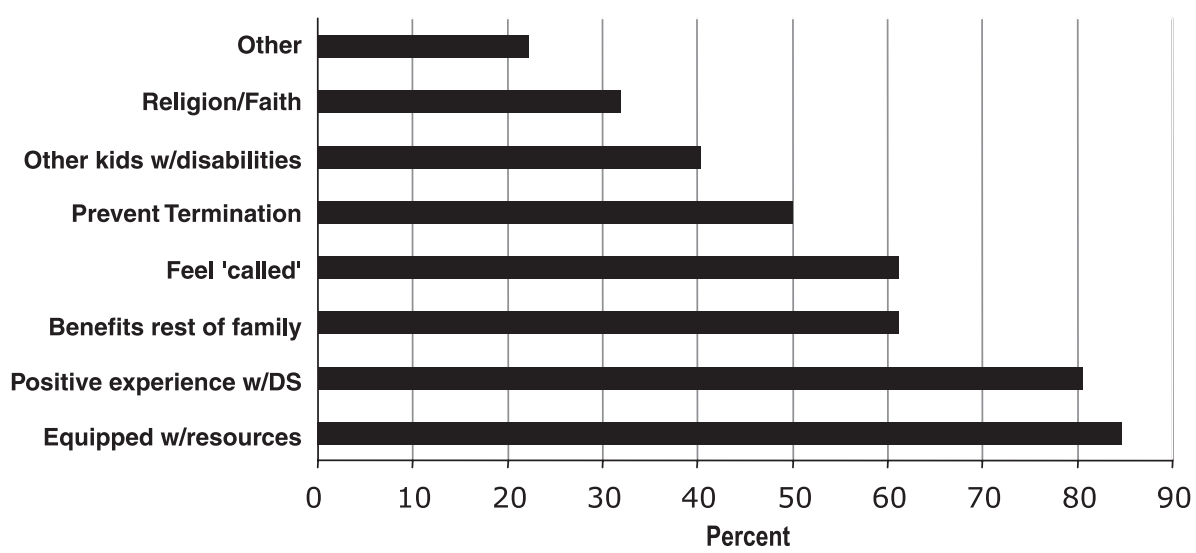

Fig. 4. Reasons people are interested in adopting a child with Down syndrome. DS, Down syndrome.

society and deserve the same chance as children without Down syndrome (93\% and $99 \%$, respectively). Although more than $90 \%$ strongly agree that adopting a child with Down syndrome would enhance their life, more than $60 \%$ also agree that this will make their life more stressful. Most respondents (84.1\%) would at least somewhat agree that it takes a special type of person to adopt a child with Down syndrome. Less than half of respondents thought that they would be helping society by adopting a child with Down syndrome, and only $6 \%$ agreed that they feel obligated to adopt a child with Down syndrome.

When asked about their perceptions regarding societal and medical professional views on individuals with Down syndrome, $70 \%$ of respondents thought that society's perception of individuals with Down syndrome was somewhat negative to overly negative. Sixty-seven percent thought that obstetricians' perception of individuals with Down syndrome was somewhat negative to overly negative, whereas $44 \%$ thought that pediatricians' views were somewhat negative to overly negative. Forty-one percent of respondents were unsure of genetic counselors' views of individuals with Down syndrome.

\section{DISCUSSION}

This study was performed to investigate the nature of families and individuals waiting to adopt a child with Down syndrome. This type of questionnaire, using a quantitative approach, is effective for measuring aspects of a wide range of genetic issues. ${ }^{9}$ On the basis of information collected from 72 families or individuals who are currently waiting to adopt a child with Down syndrome, some basic conclusions can be drawn. 
Families and individuals waiting to adopt a child with Down syndrome are committed to adoption of children not only with Down syndrome but also with disabilities in general. Approximately two thirds of respondents indicated that they had a child with a disability other than Down syndrome, approximately half had already adopted a child with a disability other than Down syndrome, and approximately one third had already adopted a child with Down syndrome. A substantial number of potential adoptive parents are currently on other waiting lists to adopt a child with Down syndrome. When specifically asked if they would rather adopt a child with or without Down syndrome, none of the respondents stated that they would rather adopt a child without Down syndrome. The length of time on the DSAGC waiting list to adopt a child with Down syndrome was between 7 months and 5 years for $47 \%$ of the respondents. The most frequent response was between 1 and 2 years. For children without special needs (including foreign adoptions), the waiting time is approximately 1 year. This indicates that the list of parents interested in adopting a child with Down syndrome greatly exceeds the availability of children with Down syndrome who are being placed for adoption. In short, there is an ample supply of parents waiting to adopt a child with Down syndrome.

The majority of families and individuals waiting to adopt a child with Down syndrome has had significant personal exposure to individuals with Down syndrome and are aware of the medical issues associated with this condition. Less than $10 \%$ of respondents had no personal exposure to individuals with Down syndrome. Given the fact that exposure to individuals with Down syndrome was so high, it is not surprising that the respondents were knowledgeable about the nature of Down syndrome and the medical conditions associated with this condition. All respondents were aware that mental retardation is a feature of Down syndrome, and that the intelligence quotient can range from mild to severe mental retardation. This study found that the majority of families and individuals waiting to adopt a child with Down syndrome were also well aware that individuals with Down syndrome have a host of other significant medical conditions.

It is not clear why more than $80 \%$ of respondents resided in nonurban communities with populations less than 200,000. Perhaps these environments are more conducive to accepting a child with special needs, whereas introducing a child with Down syndrome into an urban population may not be as easily accomplished. Another speculation would be that individuals working in urban areas may think that the pressures of daily life may impair their ability to devote sufficient care to a child with Down syndrome. The information on location of residence may also be specific to the Cincinnati program. There are numerous other agencies that are involved in placing children with Down syndrome for adoption. Results from these other agencies may differ from that found in the data gathered from the DSAGC.

One of the primary goals of this study was to identify the reasons people choose to adopt a child with Down syndrome. The responses indicated that the primary reason is that people feel equipped with the resources to raise a child with Down syndrome. On the basis of this response, it is obvious that these individuals are aware that raising a child with Down syndrome may involve the use of additional resources needed for raising a child with special needs. This is an important finding as it highlights the fact that these adoptive parents are not naive to the potential challenges of raising a child with a disability.

The second most common reason for choosing to adopt a child with Down syndrome was a previous positive experience with an individual with Down syndrome. The fact that these prospective adoptive families have had positive, firsthand experience with individuals with Down syndrome is undoubtedly a major factor in their decision to seek adoption.

Another common reason for choosing to adopt a child with Down syndrome is the belief that this choice will benefit the rest of their family. This view is in contrast with the prevailing views of several decades ago, when studies indicated that having a child with mental retardation in the family was crisisproducing, often inducing lifelong stress and distress. ${ }^{10}$ More recently, investigators concluded that adoption of a child with mental retardation may have a positive family impact. According to Glidden et al., ${ }^{11}$ the measures used to assess maternal and family adjustment to the adoption of a child with mental retardation indicated overwhelmingly positive effects, with only occasional reports of negative impact. Another investigation corroborated this observation. ${ }^{12} \mathrm{~A}$ wide range of emotions and reactions, most of them favorable, developed among siblings of children with Down syndrome. These siblings were generally welcomed into the disability community. Most siblings found rich value in having a family member with Down syndrome. This information may be contrary to the expectations of parents informed prenatally that their fetus has Down syndrome. Therefore, at the time of genetic counseling after the diagnosis of trisomy 21, this information should be discussed. It is ironic that many families choose to terminate pregnancies affected with Down syndrome because they believe that a child with mental retardation and special needs will adversely impact family dynamics, especially other children in the home, whereas families waiting to adopt a child with Down syndrome are confident that having a child with special needs will only enhance their family's cohesiveness.

Approximately half of respondents stated that choosing to adopt may prevent the termination of a fetus with Down syndrome. The pregnancy termination rate for Down syndromeaffected fetuses has been reported to be as high as $87 \% .{ }^{13}$ However, this is a selected group because many parents who would not consider termination of Down syndrome pregnancies frequently do not avail themselves of chorionic villus sampling or amniocentesis. It is not known whether adoption was offered as an option to parents informed that their fetus has Down syndrome before their decision to terminate their pregnancy. Similarly, it is not known whether offering the option of placing a child for adoption will affect the pregnancy termination rate. For parents who elect to continue their pregnancy with Down syndrome, numerous recommendations for improving their obstetric experience have been made. Among a series of 
141 women who received a prenatal diagnosis of Down syndrome and elected to continue their pregnancy, the majority expressed frustration with the care they subsequently received. ${ }^{14}$ The most common suggestions these women made were that the diagnosis be conveyed in person, that up-to-date printed materials on Down syndrome be provided, and that referral to a local Down syndrome support group be made.

Families and individuals waiting to adopt a child with Down syndrome believe that individuals with Down syndrome are an important part of society. From this questionnaire, it is apparent that prospective adoptive families understand the challenges of raising a child with Down syndrome. In fact, more than half agreed that raising a child with Down syndrome would make their life more stressful.

In general, respondents thought that society as a whole had a negative perception of individuals with Down syndrome. Their perceptions of beliefs within the medical profession varied. However, the majority strongly agreed that awareness about the option of adoption for babies with Down syndrome needed to be increased within the medical community.

There are some limitations to this study. On the basis of the response rate of $36.2 \%$ for families and individuals waiting to adopt a child with Down syndrome, it is not possible to determine whether the respondents differ in important ways from nonrespondents. Specific mention was made in the cover letter that these surveys would be anonymous and in no way would their participation or answers affect their standing with the DSAGC or the Adoption Awareness Program. However, one cannot be sure that certain questions in this survey may have been influenced by the relationship between the respondent and the DSAGC because of the emotional investment involved in waiting to adopt and the desire to be selected to receive a child with Down syndrome for adoption.

Data were unavailable regarding the reasons the potential families were so positive and thought they had the resources to meet the needs for raising a child with Down syndrome. An area of future research would be to elucidate exactly what resources these individuals and families believe they have available to them that better equip them to raise a child with special needs. It would also be interesting to further assess the positive experiences adoptive parents have had with individuals with Down syndrome.

In the United States, approximately 5500 individuals are born with Down syndrome each year. ${ }^{15}$ Despite improved prenatal diagnosis and termination of affected fetuses by many couples, the birth prevalence for this condition remains significant at 1.3 per 1000 live births. ${ }^{15}$ For the majority of these families, the diagnosis of Down syndrome is unsuspected and the child is identified as having this condition only after birth. Once this diagnosis has been confirmed, it may be appropriate in some families to raise the option of placing their child with Down syndrome for adoption so that the parents can be made aware of this possibility. This discussion must be handled with sensitivity and only at an appropriate point once the parents have recovered from the emotional impact of the diagnosis. Balanced information that is accurate, current, and respectful of individuals with Down syndrome should be provided.

Families learning that their fetus has Down syndrome during pregnancy are often devastated by this news. They have a difficult decision to make, usually in a short period of time. A balanced approach, discussing the options of pregnancy continuation, termination, and continuing the pregnancy with the intent to place the child for adoption, should be provided. It is estimated that each year in the United States, approximately 750 women who are informed their fetus has Down syndrome elect to continue their pregnancy. ${ }^{14}$ It is not known whether being informed of the option of continuing their pregnancy with the plan to place their child with Down syndrome for adoption will affect the pregnancy termination rate. However, we believe information on the availability of adoption should be conveyed to parents in this situation so that all options are clearly known and they can make an informed decision regarding pregnancy management.

\section{ACKNOWLEDGMENTS}

This study could not have been completed without the permission and assistance of the Down Syndrome Association of Greater Cincinnati and the families who participated in this investigation.

\section{References}

1. Kornitzer M. Child Adoption in the Modern World. London: Putnam; 1952:199-214.

2. National Committee for Adoption. Adoption Factbook: United States Data, Issues, Regulations and Resources. Washington, DC: 1985.

3. National Council for Adoption. State, National and International Data on Adoption. In: Adoption Factbook III. Marshner. C, Pierce WL (Eds.). Waite Park, MN Park Press Quality Printing; 1999:27.

4. Jacobson CB, Barter RH. Intrauterine diagnosis and management of genetic defects. Am J Obstet Gynecol 1967;99:796-805.

5. Steele MW, Breg WR. Chromosome analysis of human amniotic-fluid cells. Lancet 1966;1:383-385.

6. Finnegan J. Relinquishing a child for special needs adoption. Prenat Diagn 1991;6 (1):36-38.

7. Annick-Camille D, De Vigan C, Julian-Reynier C, Goujard J, et al. Adoption and fostering of babies with Down syndrome: a cohort of 593 cases. Prenat Diagn 1998; 18:437-445.

8. Linforth H, Fletcher HG. New families for Down's children. Br J Hosp Med 1989;42 (5):427-435.

9. Beeson D. Nuance, complexity, and context: qualitative methods in genetic counseling research. J Genet Couns 1997;6:21-43.

10. Solnit AJ, Stark MH. Mourning and the birth of a defective child. Psychoanal Study Child 1961;16:523-537.

11. Glidden LM, Malliere VN, Herbert SL. Adopted children with mental retardation: positive family impact. Ment Retard 1988;26 (3):119-125.

12. Skotko BG, Levine SP. What other children are thinking: brothers and sisters of persons with Down syndrome. Am J Med Genet C Semin Med Genet 2006;142 (3): $180-186$.

13. Kramer RL, Jarve RK, Yaron Y, MP, Johnson et al. Determinants of parental decisions after the prenatal diagnosis of Down syndrome. Am J Med Genet 1998;79: $172-174$.

14. Skotko BG. Prenatally diagnosed Down syndrome: mothers who continued their pregnancies evaluate their health care providers. Am J Obstet Gynecol 2005;192: $670-677$.

15. Centers for Disease Control and Prevention (CDC). Improved national prevalence estimates for 18 selected major birth defects-United States, 1999-2001. MMWR Morb Mortal Wkly Rep 2006;54:1301-1305. 